\section{¿ESTAMOS ASISTIENDO A UNA ERA DE TEORIZACIÓN DE LA BIOLOGÍA?}

\author{
Juan J. L. Velázquez \\ Instituto de Ciencias Matemáticas \\ ICMAT (CSIC-UAM-UC3M-UCM) \\ Facultad de Matemásticas \\ Universidad Complutense \\ Madrid 28040. España \\ jj_velazquez@icmat.es
}

\begin{abstract}
During the last times there has been an increasing interest by physicists and mathematicians in the study of problems allowing to understand biological questions. On the other hand, progress in experimental techniques are making possible to obtain a huge amound of information about the mechanisms used by cells. In this article some mathematical research lines whose study has been motivated by biological problems are described.
\end{abstract}

KEY WORDS: Theoretical biology; stochasticity; pattern formation; kinetic models.

Durante la última década se han incrementado considerablemente las interacciones entre biólogos y matemáticos. El número de participantes en las reuniones científicas dedicadas a la biología teórica o a biología matemática ha crecido exponencialmente durante los últimos años.

Existen varias razones que explican el enorme desarrollo que ha experimentado en los últimos años este área de investigación. Posiblemente la más importante sea el gran desarrollo que han experimentado las técnicas experimentales que se aplican en la biología durante las últimas décadas. En el último medio siglo se ha logrado desentrañar la estructura detallada de numerosas "máquinas biológicas". En la actualidad resulta también posible seguir con un considerable grado de detalle la dinámica de numerosas biomoléculas que juegan un papel esencial en los organismos vivientes. Por otra parte se ha obtenido el genoma completo de varios organismos y se está comenzando a obtener una gran cantidad de información cuantitativa sobre numerosos sistemas moleculares de la biología.

\section{ARE WE IN THE TIMES OF THEORETICAL BIOLOGY?}

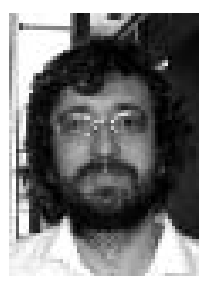

RESUMEN: Durante los últimos años ha habido un creciente interés por parte de físicos y matemáticos en el estudio de problemas que surgen al tratar de comprender cuestiones de biología. Por otra parte los avances en las técnicas experimentales están permitiendo obtener una gran cantidad de información sobre los mecanismos que emplean las células en su funcionamiento. En este artículo se describen algunas líneas de investigación en matemáticas cuyo estudio ha sido motivado por el estudio de problemas biológicos.

PALABRAS CLAVE: Biología teórica; estocasticidad; formación de patrones; modelos cinéticos.

Esta explosión de datos experimentales ha despertado un gran interés entre numerosos físicos y matemáticos. Estas comunidades cientificas llevan empleando sofisticadas herramientas matemáticas desde hace generaciones y son conscientes de lo mucho que dichas técnicas han contribuido para mejorar la comprensión que tenemos en la actualidad de los problemas físicos y químicos. Muchas de las leyes fundamentales de estas ciencias no pueden ni siquiera formularse sin emplear un lenguaje matemático bastante sofisticado. ¿Podría haber nuevas leyes científicas, que están aún por descubrir y a las que sólo se podría acceder repensando los problemas biológicos con la precisión y el grado de formalización que emplean habitualmente los científicos que trabajan en las ciencias exactas?

En las siguientes páginas se describirán algunas pocas áreas concretas en las que físicos y matemáticos están tratando de emplear su forma de abordar los problemas para tratar de comprender mejor los problemas biológicos. Estas áreas no son ni muchos menos las únicas. En la actualidad hay una enorme cantidad de investigadores, procedentes de 
casi todas las especialidades de las matemáticas que están repensando numerosos problemas biológicos. Tampoco todas las áreas de la biología que están interaccionando en la actualidad con las matemáticas son las que se mencionan en este artículo. El tipo de cuestiones que se mencionarán son principalmente el tipo de problemas que están interesando a matemáticos y físicos habituados a trabajar con ecuaciones diferenciales.

\section{La RELACIÓN ENTRE BIOLOGÍA Y MATEMÁtICAS ES BASTANTE MÁS ANTIGUA DE LO QUE A VECES SE DICE...}

La relación entre las matemáticas y la biología no es nueva en absoluto. Tampoco lo es el área de conocimiento que se suele denominar Biología Teórica. Es interesante observar que E. Schrödinger ya se preguntaba en 1944 si para comprender sistemas biológicos bastaría con emplear las leyes físicas descubiertas hasta entonces o si sería necesario hallar nuevas leyes que fuesen aplicables a sistemas que tienen la peculiar organización que tienen los átomos y moléculas de los sistemas biológicos. Como también observaba E. Schrödinger las leyes y regularidades de la física se han obtenido en numerosos casos estudiando materiales inorgánicos cuya estructura es completamente distinta de la de los sistemas biológicos (cf. [26]).

El estudio de problemas biológicos estimuló y promovió el descubrimiento de muchas de las leyes fundamentales de la Mecánica. Esto incluye algunas de las primeras investigaciones sobre resistencia de materiales, el estudio de los flujos viscosos en tubos o el principio de conservación de la energía (cf. [14]).

Por ejemplo, Galileo formuló algunos argumentos de análisis dimensional en los que mostraba que las dimensiones de los organismos biológicos no pueden crecer de forma arbitraria debido a los límites impuestos por la resistencia de los materiales de los que estan formados los seres vivos.

Es Ilamativo que existan conexiones entre la biología y la teoría de números. Leonardo da Vinci o Goethe se interesaron por el problema de la filotaxis, es decir, en el estudio de la disposición geométrica de las algunas estructuras de las plantas, tales como ramas, semillas y otras semejantes. Hace siglos que se observó que en muchas plantas dichas estructuras se sitúan en ciertos puntos que están contenidos en unas curvas espirales, y que el número de dichas curvas, que puede variar de unas partes de la planta a otras, en muchas plantas es un número de la sucesión de Fibonacci $\{1,2,3,5,8,13,21,34,55,89 \ldots\}$. Estas espirales son particularmente visibles, por ejemplo en los girasoles o en las piñas. Una explicación convincente de este fenómeno se ha obtenido muy recientemente (cf. [10]). Los autores de este trabajo demostraron que la sucesión de Fibonacci aparece espontáneamente en algunos sistemas dinámicos que simulan la forma en la que se sitúan, por ejemplo, las semillas de las plantas. Más aún, dichos autores obtuvieron sistemas puramente mecánicos, sin relación ninguna con la biología en las que un conjunto de gotas fluidas se situaban en estructuras espirales análogas a las observadas en las plantas.

Una recopilación de muchas de las investigaciones realizadas hasta el primer tercio del siglo XX tratando de explicar las regularidades biológicas con ayuda de argumentos matemáticos se puede encontrar en el famoso libro [8]. Algunos de los muchos problemas que se estudian en dicho libro son, por ejemplo, el estudio de las formas de algunos organismos sencillos (y pequeños) en los que las fuerzas de tensión superficial juegan un papel dominante, la descripción de las formas espirales que aparecen en numerosos organismos tales como los moluscos, la comparación de las diferentes teorías postuladas para explicar la formación de las colmenas de las abejas. En este interesante ejemplo se forman estructuras con una simetría hexagonal global que aparece a partir de la interacción de una gran cantidad de dinámicas individuales que por sí mismas no darían lugar a dicha simetría. En [8] se estudia también el empaquetamiento óptimo de células individuales sometidas a fuerzas de presión externa, etc., la ley de Murray que describe la ley de potencias a la que deben de ajustarse los radios de las arterias y capilares para obtener la máxima eficiencia en el transporte del flujo sanguíneo en los animales, etc.

El libro [8] es una interesante muestra de la gran cantidad de problemas biológicos que habían sido estudiados usando matemáticas ya en el siglo XIX y comienzos del $X X$. Una gran parte del libro examina el efecto que tienen las leyes de la mecánica en las formas de los organismos 
biológicos. No es por ello casual que el área de la biología en donde posiblemente existe un mayor grado de matematización sea la biomecánica, un tema al que me referiré con más detalle posteriormente.

Hay otras muchas áreas de biología que han tenido interacciones relevantes con la matemática en el pasado. En particular, no ha sido inusual que el estudio de problemas matemáticos motivados por la biología haya producido progresos relevantes en matemáticas. El estudio de la genética de poblaciones estuvo en el origen muchos importantes avances en estadística obtenidos por R. A. Fisher. El estudio de la dinámica de poblaciones ha producido interesantes avances en la teoría de ecuaciones diferenciales ordinarias. A. M. Turing descubrió una famosa inestabilidad que lleva su nombre (cf. [29]) que puede tener lugar en los sistemas de ecuaciones diferenciales que describen reacciones químicas combinadas con procesos de difusión tal y como sucede frecuentemente en sistemas biológicos. El estudio del sistema de ecuaciones formulado por HodkingHuxley para describir la propagación de señales químicas y eléctricas en el axón del calamar gigante ha dado lugar al desarrollo de diferentes técnicas para el estudio de ecuaciones en derivadas parciales...

\section{Estocasticidad: Procesos con pocas moléculas}

Numerosos procesos biológicos tienen lugar en regiones de dimensiones tan pequeñas que los efectos de las moléculas individuales juegan un papel esencial.

Consideremos por ejemplo el caso de E. coli. Esta bacteria es uno de los "organismos modelo" que han sido estudiados por los biólogos con gran detalle. En particular, un proceso biológico para el que se puede obtener información a partir del estudio de este organismo es el de la movilidad celular.

La bacteria $E$. coli se mueve mediante la propulsión que le suministra un conjunto de flagelos (entre 4 y 10) situados en la parte posterior de la célula. Entender la forma detallada en la que el movimiento flagelar produce la fuerza de propulsión que mueve a la bacteria es una cuestión interesante de mecánica de fluidos que ha recibido una considerable atención en el pasado y que fue resuelta hace varias décadas. Un aspecto esencial de este problema es que, debido a la pequeñez de la bacteria, su movimiento tiene lugar a números de Reynolds muy pequeños, o dicho en términos menos técnicos, las fuerzas de viscosidad del fluido son mucho más importantes que la inercia del movimiento. Resultados clásicos de mecánica de fluidos, que pueden encontrarse en los libros de texto, establecen que la fuerza de fricción, cuando el número de Reynolds es muy pequeño, es proporcional a la velocidad, y es mayor para un cilindro que se mueve perpendicularmente a su eje que para un cilindro que se mueve paralelamente a su eje. Cuando los flagelos producen el desplazamiento de la bacteria tienen una forma aproximadamente helicoidal en la parte posterior de la bacteria, y realizan un movimiento de rotación en torno al eje de dicha hélice. Es fácil ver que en ese movimiento los flagelos no se mueven con relación al fluido ni paralelamente ni perpendicularmente a su eje, sino con una combinación de ambos movimientos. La diferencia de fuerzas de fricción para estos tipos de movimiento que fue mencionada anteriormente tiene como consecuencia que la fuerza neta experimentada por el flagelo no sea opuesta al movimiento de rotación del flagelo, sino que tiene una componente adicional en la dirección hacia la que esta orientada la célula. Dicha componente adicional es la que proporciona la propulsión celular. Una descripción más detallada de este mecanismo se encuentra en [5], [30].

El efecto de la estocasticidad que se mencionaba en el título de esta sección no aparece, sin embargo, en el estudio de la propulsión mediante flagelos, que es un problema que puede estudiarse en gran parte mediante las ecuaciones clásicas de la mecánica de fluidos, sino en otra parte del problema, concretamente en la detección de las señales que indican a la célula la dirección hacia la que debe moverse. Los flagelos tienen dos formas distintas de moverse. Puede ocurrir que todos los flagelos roten de forma coordinada, o por el contrario puede suceder que los flagelos se muevan de forma independendiente y descoordinada. En el primer caso, la célula se mueve en forma aproximadamente rectilinea y se suele decir que la bacteria "corre". En el segundo caso la bacteria se mueve de forma esencialmente aleatoria, sin ninguna orientación definida y se suele decir que "da tumbos". El que la bacteria se mueva de una u otra forma depende del grado de ocupación de unos receptores químicos situados en la superficie de la célula. Dichos receptores miden la 
concentración externa a la célula de ciertas sustancias químicas que la bacteria necesita para sobrevivir. La más estudiada de estas sustancias se denomina aspartato. Es precisamente en este proceso de medición donde aparecen los efectos de estocasticidad. En efecto, las bacterias de E. coli son muy pequeñas (su longitud es de $2 \times 10^{-4}$ $\mathrm{cm}$ ) y por ello el número de receptores no es demasiado grande, a lo sumo de unos pocos centenares. La bacteria tiene tendencia a moverse hacia las regiones con mayor concentración de aspartato y para ello la estrategia que sigue es la de incrementar el número de "carreras" frente a "tumbos" cuando percibe que los receptores incrementan su grado de ocupación. Sin embargo, debido a la pequeñez del número de receptores, el grado de ocupación de los receptores no es una variable determinista, sino una variable aleatoria, en la que las fluctuaciones en torno al valor medio no son despreciables. Esta estocasticidad se manifiesta en el hecho de que muchas de las variables que harian falta para describir la dinámica de las bacterias de E. coli, como por ejemplo la distancia recorrida en cada "carrera" o la duración de las mismas, son variables aleatorias.

Esta estocasticidad, inherente a la dinámica de numerosos organismos, es bastante habitual en los procesos biológicos y se debe a las pequeñas dimensiones de estos sistemas. El mundo en que viven muchos de estos organismos no es un mundo determinista, newtoniano, que pueda describirse mediante ecuaciones diferenciales. Por el contrario, las bacterias y muchas otras estructuras biológicas han aprendido a usar la aleatoriedad para sobrevivir. Desde el punto de vista del matemático esto plantea interesantes cuestiones y retos a la hora de obtener modelos y describir la dinámica de estos organismos.

Aunque las ecuaciones de los procesos estocásticos que rigen la química de sistemas con un número reducido de moléculas se conocen desde hace bastante tiempo (cf. [16]), la descripción de las soluciones de dichas ecuaciones, sobre todo en sistemas que contienen un gran número de especies moléculares distintas es un reto matemático de gran envergadura que presenta considerables dificultades tanto desde el punto de vista analítico como numérico. El estudio de las propiedades matemáticas de los procesos estocásticos que describen reacciones químicas que involucran a una cantidad pequeña de moléculas es uno de los temas que está experimentando un importante desarrollo en la actualidad (cf. [11], [12]).

\section{Cálculo de propiedades efectivas de sistemas BIOLÓGICOS FORMADOS POR NUMEROSAS PIEZAS SIMILARES}

Son innumerables las estructuras biológicas que están formadas por un elemento que se repite un gran número de veces, pero en donde la estructura actúa de forma colectiva mediante la acción coordinada de las diferentes piezas individuales. Piénsese por ejemplo en los estomas de las hojas de las plantas, los conos y bastones que actúan como receptores de la luz en la retina, la estructura celular de los tejidos, los cilios que propulsan a muchas células...

Durante los últimos cincuenta años los matemáticos han desarrollado un conjunto de técnicas para estudiar el comportamiento colectivo de sistemas formados por múltiples piezas individuales que se conocen con el nombre de métodos de homogenización. Dichos métodos se han aplicado con éxito en el estudio de las propiedades de medios materiales, y recientemente han comenzado a ser usados en el estudio de alguno de los sistemas biológicos mencionados arriba (cf. [3]).

Un ejemplo de sistema biológico que puede estudiarse empleando las ideas de la teoría de homogenización, que es particularmente atractivo por varias razones, es el estudio de la forma en la que los receptores de una célula capturan una sustancia química. Es bastante frecuente que una célula que precisa obtener un tipo específico de molécula que se encuentra distribuida en su entorno disponga de un conjunto de receptores, que típicamente son otra molécula o un grupo molecular, y que tienen una gran afinidad química por la molécula que se pretende capturar. ¿Cuál es la disposición y el número óptimo de receptores que permiten atrapar la sustancia química externa de la forma más eficaz posible? La respuesta a este problema tal y como está planteado sería la de recubrir toda la superficie celular de receptores, pero en ese caso quedaría poco espacio para otras estructuras. ¿Es posible conseguir un grado de eficiencia razonable en la captura de sustancias externas sin recubrir toda la superficie celular? La solución de este problema puede encontrarse en [5] en donde se estudia esta cuestión suponiendo que la célula es esférica. La geometría no esférica puede estudiarse de forma similar, incrementando algo las dificultades técnicas, pero no el resultado esencial. El análisis de [5] llega a una conclusión interesante. Supongamos que el radio de la célula es $R$ y 
que el tamaño de cada receptor es a. Típicamente a es mucho menor que $R$. Supongamos también que se tienen $N$ receptores en la superficie de la célula. En principio podría pensarse que para conseguir que la captura de la sustancia externa ocurra con la misma rapidez con la que sucedería si toda la superficie externa estuviese llena de receptores haria falta que una fracción importante del área de la célula estuviese llena de receptores, es decir que se tuviese

$$
N\left(\frac{a}{R}\right)^{2} \approx 1
$$

Lo sorprendente, es que dicha tasa óptima de captura puede obtenerse con un número mucho menor de receptores, concretamente si

$$
N\left(\frac{a}{R}\right) \approx 1
$$

La razón por la que sucede esto es porque antes de ser capturada por los receptores las moléculas de la sustancia externa se mueven de forma browniana por el exterior de la célula, lo que es un método muy eficaz de explorar la superficie celular.

Algo que es particularmente llamativo en este ejemplo es el hecho de que el límite (1) es precisamente el que da lugar a problemas matemáticos más interesantes cuando el problema se formula en términos de ecuaciones diferenciales. Por interesante se entiende aquí que en ese régimen los receptores comienzan a actúar de forma colectiva y no pueden despreciarse la interacción entre ellos. Esta capacidad de los sistemas biológicos para "identificar" límites matemáticos relevantes ocurre con alguna frecuencia. Otro ejemplo en el que también sucede esto es en el problema del movimiento de la bacteria $E$. coli que se mencionó en el apartado anterior. En ese problema el tiempo medio de duración de las "carreras" es exactamente del orden de magnitud necesario para que los efectos del movimiento browniano de la bacteria comiencen a ser importantes. Si dicho tiempo hubiese sido mayor las bacterias tendrian un movimiento puramente difusivo y serían incapaces de dirigirse hacia las regiones con mayor concentración de aspartato. Un tiempo menor tendría como consecuencia que la velocidad media hacia las regiones con altas concentra- ciones de aspartato sería menor. Presumiblemente la actuación de la evolución darwiniana tratando de lograr una mayor eficiencia ha sido la responsable de estos delicados ajustes que posiblemente van a plantear interesantes problemas a los matemáticos en los próximos años.

Otro tipo de problemas biológicos en los que los métodos de la teoría de homogenización y otros relacionados podrían jugar un papel interesante es en el estudio de las ecuaciones efectivas de los medios aleatorios. Un ejemplo reciente en el que se esbozan nuevas herramientas matemáticas para estudiar problemas biológicos se puede ver en [6]. Numerosas estructuras biológicas están formadas por células que tienen un importante grado de variabilidad y que han de ser descritas mediante variables estocásticas. La teoría de los procesos difusivos en medios aleatorios ha sido desarrollada en gran parte por matemáticos puros interesados en la teoría de la probabilidad, y por físicos interesados en cuestiones tales como difusión de neutrones o problemas análogos. Sin embargo, con bastante frecuencia los problemas que se han abordado en dichas áreas no son exactamente las cuestiones que surgen en los problemas biológicos. En otros casos, en particular en el caso de los resultados obtenidos por los matemáticos más teóricos, el lenguaje en el que han sido formulados los resultados plantea serias dificultades a los científicos que se dedican a la biología.

\section{TeORÍA CINÉtICA dE LOS SISTEMAS BIOLÓGICOS}

Un tema en el que en los últimos años ha habido una gran actividad por parte de varios grupos de matemáticos y físicos ha sido en el estudio de ecuaciones cinéticas para describir agregados celulares. Matemáticamente dichas ecuaciones son bastante similares a las que se emplean en la dinámica de gases enrarecidos. La idea que subyace bajo estos modelos es la de considerar a las células como si fuesen las "moléculas" de un gas. En los modelos dichas células cambian su dirección de movimiento o su velocidad o bien de forma aleatoria, o bien debido a la interacción con otras células. La idea de describir de esta forma los agregados celulares no es nueva (cf. [1], [23]), aunque en los últimos años se han realizado progresos en el estudio matemático de alguno de estos modelos. Una de las aplicaciones más habituales de estos estudios es la de obtener 
"coeficientes de difusión" para las células de la misma forma en que las ecuaciones cinéticas de la dinámica de gases permiten obtener valores de los coeficientes de difusión, viscosidad y otros análogos (cf. [9]).

En la mayor parte de los modelos cinéticos de células que se han estudiado hasta ahora se suele suponer que las células no interaccionan con su entorno, excepto por la interacción indirecta que las células puedan tener con una sustancia química que ellas mismas producen. Uno de los pocos modelos cinéticos en donde se tienen en cuenta las interacciones entre células es el estudiado en [15]. La ecuación cinética considerada en dicho artículo describe la evolución de un agregado celular bidimensional formado por células que se caracterizan mediante dos variables individuales, que son su posición y su orientación. Cuando dos células del agregado se encuentran, se reorientan y tratan de alinearse en la misma dirección. Desde el punto de vista matemático estas ecuaciones tienen considerables analogías con la ecuación de Boltzmann que se emplea en el estudio de la dinámica de gases, aunque existen también algunas diferencias. En efecto, en la ecuación de Boltzmann dos partículas que se encuentran no tratan de alinear su velocidad, sino que colisionan elásticamente. En concreto las soluciones de la ecuación de Boltzmann satisfacen principios de conservación de la energía y de la cantidad de movimiento, que no se cumplen para las ecuaciones de [15]. Como consecuencia, el tipo de dinámicas que se obtienen para estas últimas ecuaciones es bastante distinto del que se obtiene en la dinámica de gases. En el caso de la ecuación considerada en [15] existen soluciones en las que las células se alinean en dos direcciones opuestas, un tipo de comportamiento que se ha observado en unos organismos llamados myxobacterias, que se han estudiado de forma bastante detallada con el fin de obtener información sobre los mecanismos de movilidad celular (cf. [20]).

La principal objección que se puede plantear a los modelos cinéticos de agregados celulares es que, en muchos casos, las densidades celulares son demasiado altas para que estas ecuaciones puedan proporcionar una descripción realista de dichos agregados. En el caso de la dinámica de gases se conocen desde hace más de cincuenta años las hipótesis que deben satisfacer las interacciones moleculares y las densidades de partículas para que se dicha dinámica se pueda describir empleando ecuaciones cinéticas. Por ejem- plo, la ecuación de Boltzmann es válida si el camino libre medio entre colisiones es mucho mayor que la distancia de interacción mutua entre moléculas. La tarea de precisar matemáticamente las hipótesis que garanticen la validez de los límites cinéticos para agregados celulares no ha sido realizada aún. Uno de los factores que dificultan este problema es el hecho de que muchas de estas interacciones tienen lugar mediante la emisión de sustancias químicas que pueden tener sus propias longitudes y tiempos característicos asociados tales como el tiempo medio de degradación, la longitud característica de difusión y otros. Posiblemente, uno de los pocos ejemplos experimentales en donde la distancia entre las bacterias es lo suficientemente grande como para que puedan usarse modelos cinéticos que den información cuantitativa sobre las densidades de agregados celulares es el considerado en [19].

\section{Movilidad celular y biomecánica}

Muchos de los problemas mencionados hasta ahora están relacionados con la movilidad celular. El movimiento de las células juega un papel importante en una gran cantidad de procesos biológicos tales como embriogénesis, inflamación, curación de heridas... Por otra parte el estudio de los sistemas que emplean las células para desplazarse plantea una gran cantidad de cuestiones físicas y matemáticas de interés.

Se han descrito anteriormente algunos de los problemas de mecánica de fluidos que plantea el desplazamiento empleando flagelos de algunos organismos tales como E. coli. Tal y como se indicó allí, el origen de la fuerza producida por los flagelos se entiende bien. Algo que sin embargo no se comprende de forma tan satisfactoria es la forma en la que los flagelos se reorganizan con el fin de rotar conjuntamente en forma coordinada. Se sabe que dicha reorganización sucede como respuesta a la saturación de los receptores que miden la concentración de aspartato en la superficie celular, pero no se conoce el mecanismo detallado, que posiblemente involucra, además de a las señales químicas, las propiedades mecánicas del flagelo y del medio fluido que lo rodea.

Otro tipo de propagación celular, diferente de la producida por los flagelos, es la propagación ameboide, debida a la 
expansión y retracción de pseudópodos que tiene lugar en algunas células eucariotas como por ejemplo en el "organismo modelo" Dictyostelium discoideum (Dd). Las fuerzas que dan lugar al movimiento de este tipo de organismos se deben principalmente a la dinámica de una estructura interna a la célula denominada citoesqueleto que además de estas funciones motrices desempeña otras muchas funciones importantes para la vida celular, tales como transportar sustancias quimicas de unas partes de la célula a otras. El citoesqueleto es una estructura muy dinámica y cuya estructura puede variar dependiendo del grado de ocupación de algunos receptores externos a la célula que permiten medir, por ejemplo, gradientes de sustancias químicas. Otro factor que también juega un papel importante en el movimiento de este tipo de células son las moléculas que adhieren el citoesqueleto con el substrato externo en el que se apoya la célula. Uno de los intentos de obtener un modelo mecánico para este tipo de sistemas se puede encontrar en [2].

En todos los ejemplos mencionados anteriormente se han descrito ejemplos de movimiento celular de células individuales. Sin embargo, un problema que tiene particular relevancia es el del estudio de sistemas biológicos en los que muchas células den lugar a movimientos colectivos, tal y como sucede por ejemplo en los procesos de embriogénesis. Desde el punto de vista matemático el problema sería el de obtener "ecuaciones efectivas" para el movimiento de agregados celulares que individualmente se mueven con los mecanismos indicados anteriormente. En este punto es relevante mencionar, aunque sea de forma fugaz el área de la biomecánica. Este área que está considerablemente desarrollada ha abordado una gran cantidad de problemas que van desde el estudio de los flujos sanguíneos en el sistema circulatorio hasta las ecuaciones constitutivas de los tejidos pasando por los mecanismos de vuelo empleados por los insectos o las aves. En la búsqueda de ecuaciones constitutivas para agregados celulares es muy posible que los matemáticos familiarizados en el uso de técnicas de homogenización pudiesen realizar alguna contribución de interés.

\section{Patrones en Biología}

Uno de los temas clásicos de biología matemática ha sido el estudio de los diversos patrones que pueden aparecer en los sistemas biológicos. En biología abundan patrones geométricos tales como las espirales, patrones en forma de diana, agregados de puntos, franjas, cuadrados y otros muchos. Algunos de los problemas más conocidos que han sido estudiados por muchos de los fundadores de la moderna biología matemática son, entre otros, el estudio de las manchas de los leopardos o las cebras, el estudio de la pigmentación de los peces, el estudio de los patrones que aparecen en la superficie de las conchas marinas 0 los patrones de ondas espirales que forman las señales químicas emitidas por el Dd.

Todas estas investigaciones han incrementado considerablemente la perspectiva de los matemáticos que han aprendido así la inmensa cantidad de comportamientos distintos a los que pueden dar lugar aparentemente sencillas ecuaciones diferenciales en derivadas parciales.

Sin embargo, en los últimos tiempos se ha ido abriendo paso la idea de que independientemente de su valor estético lo que realmente resulta relevante de los variados patrones que aparecen en sistemas biológicos es el determinar, su funcionalidad, es decir, determinar si realmente los organismos los crean porque dichos patrones son más eficaces para obtener algún beneficio desde el punto de vista biológico, o si por el contrario son simplemente un "resto de la evolución". Una segunda razón por la que resulta interesante el estudio de los patrones es porque en ocasiones proporcionan información sobre los mecanismos subyacentes de interacción celular. En muchas ocasiones los organismos muestran sorprendentes patrones geométricos pero que sólo se manifiestan en condiciones muy especiales en el laboratorio. En dichos casos los patrones no tienen ningún valor biológico para el organismo pero permiten inferir, por ejemplo, que las células individuales emiten alguna sustancia que las permite dar lugar a un comportamiento colectivo. Un ejemplo interesante de esto se encuentra en [7], donde los autores obtienen tal tipo de conclusión a partir de los patrones que muestran ciertos agregados de E. coli.

Algunos de los patrones más importantes que aparecen en las estructuras biológicas son los que se manifiestan durante el desarrollo embrionario de los organismos multicelulares. En dichos procesos una cascada de reacciones químicas da lugar a los procesos de diferenciación celular responsables de que cuando el organismo esté completa- 
mente formado, cada célula esté situada en el lugar exacto en el que desempeñará precisamente la función biológica para la que está adaptada. Hay una cantidad enorme disponible de información sobre las señales químicas que se transmiten durante estos procesos (cf. [21]).

Los patrones que aparecen durante la formación y el desarrollo de los organismos han sido estudiados en numerosas especies. Dos de los más conocidos son la mosca del vinagre (Drosophila) y el metazoo conocido como Hydra. En el caso de la Drosophila hemos llegado a conocer, mediante el trabajo de cientos de investigadores de todo el mundo, los procesos químicos que se producen durante el desarrollo del organismo con un grado muy considerable de detalle. Por otra parte se ha experimentado con los patrones celulares a los que da lugar la Hydra desde hace más de dos siglos (cf. [28]). La aparente sencillez de este organismo, pero a su vez la riqueza de patrones a los que da lugar le ha convertido también en uno de los organismos preferidos de los investigadores para desarrollar modelos matemáticos que traten de explicar la gran variedad de estructuras que aparecen en los experimentos mediante principios simples (cf. [17], [18], [24]). Aunque algunos de estos modelos logran describir algunos de los efectos que se pueden ver en los experimentos, no es sin embargo tan evidente cuales son los principios que regulan la coordinación del gran número de moléculas involucradas en este tipo de procesos, ni si dichos principios generales existen. Es importante observar que dichos procesos bioquímicos que dan lugar a la formación de los patrones del desarrollo parecen ser lo suficientemente flexibles como para admitir cambios a lo largo del tiempo durante la evolución de los organismos (cf. [31]).

Muchos de los mecanismos de formación de patrones que se han estudiado en el pasado se basan en el mecanismo propuesto por Turing (cf. [29]), aunque en ellos se introducen términos no lineales que pueden dan lugar a efectos no incluidos en [29]. El mecanismo de Turing se basa en la existencia de al menos dos tipos de morfógenos en un sistema, que son un activador de corto alcance y un inhibidor de largo alcance. Dichos morfógenos se difunden en el medio y tienen la capacidad de interaccionar en puntos distintos de donde fueron producidos. Sin embargo, en numerosos sistemas biológicos las señales producidas por las células pueden permanecer en la posición donde fueron emitidas y no difundirse. En otros casos, el efecto de las células no es emitir una señal química, sino modificar el entorno en el que se encuentran dichas células por medios mecánicos. En este tipo de procesos las señales celulares no se difunden. Una clase de modelos que podrian describir la dinámica celular en presencia de señales no difusibles fue formulada en [22]. Los comportamientos de las soluciones que se tendrian en dichas situaciones se describe en [27].

\section{Robustez y Complejidad}

Los términos "robustez" y "complejidad" aparecen con gran frecuencia en la literatura biológica reciente. La definición de estos términos es aún vaga e imprecisa. Sin embargo, el tipo de cuestiones que giran habitualmente en torno a estos conceptos está atrayendo de forma creciente la atención de algunos matemáticos.

En la actualidad empiezan a conocerse con gran detalle la forma en que interaccionan las moléculas de numerosos sistemas bioquímicos importantes. Muchas de estas redes bioquímicas están formadas por un gran número de moléculas distintas. Por otra parte dichas redes son "robustas", en el sentido de que son capaces de seguir funcionando incluso aunque alguna de las sustancias que la forman no esté presente o sea modificada mediante una mutación. La "robustez" de los sistemas biológicos así entendida, le proporciona una cualidad altamente deseable, que es la capacidad de evolucionar, mediante pequeños cambios. Es bastante plausible que dicha robustez sea una consecuencia de la elevada "complejidad" de las redes.

Los conceptos de robustez y complejidad no sólo se aplican a nivel molecular, sino que también se usan frecuentemente para referirse a procesos que tienen lugar en la célula, el organismo e incluso a nivel ecológico. No existe aún una teoría clara y precisa que permita abordar este tipo de cuestiones, incluso suponiendo que fuese posible. Sin embargo, comienza a haber algunos resultados e investigaciones que sugieren cómo podría ser dicha teoría.

En los últimos años ha habido un interés creciente en lo que se suele denominar teoria de redes (cf. [4]). En el caso particular de las redes bioquímicas una cuestión que ha sido estudiada en gran detalle es la relación que existe 
entre la topología de las redes y el tipo de dinámicas a las que pueden dar lugar (cf. [13], [25]).

Por otra parte, el tipo de estructura matemática que deberían tener los sistemas biológicos para ser "robustos" en algún sentido ha sido explorado de forma detallada en [31]. La idea principal es que dichos sistemas deberían tener una gran cantidad de posibles variaciones que "funcionasen" de forma equivalente, con algunos posibles cambios bruscos, aunque raros, para algunas configuraciones específicas. Esta idea se aplica en [31] a numerosos sistemas biológicos. Posiblemente el sistema biológico en el que está idea se puede ver de forma más clara es en las diferentes realizaciones geométricas de las moléculas de ARN. Dichas moléculas pueden estar formadas por muy diferentes combinaciones de bases. Sin embargo, su función biológica parece depender principalmente de la geometría tridimensional de dichas moléculas. Teoría y experimentos muestran que configuraciones geométricas equivalentes pueden obtenerse mediante una gran cantidad de combinaciones de bases distintas. Esto explicaría por qué pequeñas mutaciones no tendrian graves consecuencias para el funcionamiento de estos sistemas, pero a su vez también explicaria que algunas mutaciones podrian generar modificaciones importantes en el funcionamiento químico de estas moléculas.

\section{8. ¿Puede la Biología dar lugar a una MATEMÁTICA COMPLETAMENTE NUEVA?}

Los ejemplos que se han mencionado anteriormente tratan de mostrar el tipo de contribuciones que las Matemáticas podrían realizar en la Biología. Sin embargo, comienza a ser cada vez más frecuente oir entre algunos matemáticos la pregunta formulada en forma inversa: ¿Qué contribución ha hecho (o podría hacer) la Biología por las Matemáticas?

Los matemáticos que formulan esta pregunta son conscientes de la enorme influencia que ha tenido la Física en el desarrollo de áreas enteras de las Matemáticas. La Mecánica Clásica, la Física Estadística, la Mecánica
Cuántica, la Relatividad General, han tenido una tremenda influencia en el desarrollo de numerosas ramas de las Ecuaciones Diferenciales, la Geometría, el Análisis Funcional o la Teoría de la Probabilidad. En los últimos años cuestiones que tienen su origen en la Física de Altas Energías están teniendo una gran influencia en el desarrollo de algunas ramas de Algebra y Geometría. ¿Podría la Biología convertirse en una fructífera fuente de cuestiones matemáticas de la misma forma que lo es la Física?

Es cierto que problemas de Biología han dado lugar ya a algunos resultados importantes de Estadística Matemática, Sistemas Dinámicos, Ecuaciones Diferenciales en Derivadas Parciales o la Teoría de Procesos Estocásticos. Sin embargo, la Biología posiblemente no ha producido aún revoluciones matemáticas como las que en su día supuso la Mecánica Cuántica o la Relatividad General. Es posible que la mayor contribución que ha realizado la Biología a las Matemáticas sea el descubrimiento de la inestabilidad de Turing, que está detrás de una gran parte de los modelos de formación de patrones que estudian físicos y matemáticos. El hallazgo de Turing es sorprendente y profundo ya que muestra, entre otras cosas, que la combinación de procesos que individualmente tienden a producir estabilidad en un sistema pueden de forma combinada dar lugar a la generación de inestabilidades con una longitud de onda característica. Sin embargo, es difícil afirmar que este descubrimiento haya dado lugar a una amplia Teoría Matemática de la misma amplitud que la que han producido las teorías físicas.

Sin embargo, tal y como indicó Schrödinger (cf. [26]) una gran parte de las leyes de la Física que conocemos han sido obtenidas estudiando sistemas muy distintos de los sistemas biológicos. De la misma forma, las matemáticas se han desarrollado en el pasado en su mayor parte estudiando problemas muy distintos de los que surgen al pensar en los organismos vivientes. Si la experiencia del pasado puede ser una guía para el futuro, es muy posible que muchas de las cuestiones que ocupen la atención de los matemáticos dentro de dos o tres décadas sean muy distintas de las actuales, y que la Biología haya jugado un papel importante decidiendo cuáles serán esas cuestiones. 


\section{REFERENCES}

1 W. Alt, "Biased random walks models for chemotaxis and related diffusion approximations", J. Math. Biol. 9, 147-177, 1980.

2 W. Alt y M. Dembo, "Cytoplasm dynamics and cell motion: Two phase flow models", Mathematical Biosciences 156, 207-228, 1999.

3 D. Andreucci, P. Bisegna, G. Caruso, H. E. Hamm y E. DiBenedetto, "Mathematical models of the spatio-temporal dynamics of second messengers in visual transduction", Biophysical J. 85, 1358-1376, 2003.

4 A.L. Barabási, Linked: The New Science of Networks, Perseus, Cambridge, MA, 2002.

5 H. C. Berg, Random walks in biology, Princeton Univ. Press, 1993.

6 T. Bollenbach, K. Kruse, P. Pantazis, M. González-Gaitán, F. Jülicher, "Robust formation of morphogen gradients", Phys. Rev. Lett. 94, 2005.

7 E. 0. Budrene y H. C. Berg, "Dynamics of formation of symmetrical patterns by chemotactic bacteria", Nature 376, 49-53, 1995.

8 D'Arcy Thompson, On Growth and Form, Cambridge University Press, 1942.

9 R. B. Dickinson y R. T. Tranquillo, "Transport equations and indices for random and biased cell migration based on single cell properties", SIAM J. Appl. Math. 55, 5, 1419-1454, 1995.

10 S. Douady y Y. Couder, "Phyllotaxis as a physical self-organized growth process", Phys. Rev. Lett. 68, 20982101, 1992.

11 R. Erban, I. G. Kevrekidis, David Adalsteinsson, Timothy C. Elston, "Gene regulatory networks: a coarse-grained, equation-free approach to multiscale computation", Journal of Chemical
Physics, Volume 124, Issue 8, 084106 , 17 pages, 2006.

12 R. Erban and J. Chapman, "Stochastic modelling of reaction-diffusion processes: algorithms for bimolecular reactions", Physical Biology, vol. 6, n. ${ }^{\circ}$ 4, 046001, 2009.

$13 \mathrm{M}$. Feinberg, "The existence and uniqueness of steady states for a class of chemical reaction networks", Arch. Rat. Mech. Anal., 132, 311-370, 1995.

14 Y. C. Fung, Biomechanics, Springer Verlag, 1984.

15 E. Geigant y M. Stoll, "Bifurcation analysis of an orientational aggregation model", J. Math. Biol. 46 (6), 537-563, 2003.

16 D. T. Gillespie, "A general method for numerically simulating the stochastic time evolution of coupled chemical reactions", J. Comput. Phys. 22: 403-34, 1976.

17 A. Marciniak-Czochra, "Receptorbased models with diffusion-driven instability for pattern formation in hydra", J. Biol. Sys. 11: 293-324, 2003.

18 H. Meinhardt, A. Gierer, "Applications of a theory of biological pattern formation based on lateral inhibition", $J$. Cell Sci. 15, 321-346, 1974.

19 N. Mittal, E. O. Budrene, M. P. Brenner y A. van Oudenaarden, "Motility of Escherilia coli cells in clusters formed by chemotactic aggregation", Proc. Nat. Acad. Sci. 100, 23, 1325913263, 2003.

20 A. Mogilner y L. Edelstein-Keshet, "Spatio-angular order in populations of self-aligning objects: formation of oriented patches", Phys. D 89, 3-4, 346-367, 1996.

21 C. Nüsslein-Volhard, Coming to Life, How genes drive development, Kales Press, 2006.
Recibido: 28 de agosto de 2010

Aceptado: 10 de septiembre de 2010 
22 H. G. Othmer and A. Stevens, "Aggregation, blow-up and collapse. The $A B C^{\prime} s$ of taxis in reinforced random walks", SIAM J. Appl. Math. 57, 4, 1044-1081, 1997.

23 C. S. Patlak, "Random walk with persistence and external bias", Bull. Math. Biophysics 15, 311-338, 1957.

24 J. A. Sherratt, P. K. Maini, W. Jäger, W. Müller, "A receptor based model for pattern formation in hydra", Forma 10, 77-95, 1995.
25 G. Shinar, U. Alon y M. Feinberg, "Sensitivity and robustness in chemical reaction networks", SIAM J. Appl. Math., vol. 69, 4, 977-998, 2009.

26 E. Schrödinger (1944): What is life?, Cambridge University Press.

27 A. Stevens y J. J. L. Velázquez, "PDEs and Non-diffusive structures", Nonlinearity, 21 T283-T289, 2008.

28 A. Trembley, Memoires pour servir à I'histoire d'un genre de polypes d'eau douce, à bras en forme de cornes, Jean and Herman Verbeek, Leiden, 1744.

29 A. M. Turing, "The chemical basis of morphogenesis", Phil. Trans. Roy. Soc., B 237 37-72, 1952.

30 S. Vogel, Life in Moving Fluids: The PhysicalBiologyofFlow, Princeton Univ. Press, 1996.

31 A. Wagner, Robustness and Evolvability in Living Systems, Princeton University Press, 2005. 